\title{
Clinical Characteristics of COVID-19
}

\author{
Hettiarachchi $\mathbf{N} \mathbf{M}^{1}$, Manilgama $\mathbf{S} \mathbf{R}^{2}$, Jayasinghe $\mathbf{I} \mathbf{K}^{2}$ \\ Journal of the Ceylon College of Physicians, 2020, 51, 14-19
}

\begin{abstract}
Coronavirus disease 2019 (COVID-19), pandemic caused by the novel coronavirus SARS-CoV-2, has caused marked morbidity and mortality globally. The clinical spectrum of COVID-19 can be classified as asymptomatic, mild, moderate and severe disease. Majority of the symptomatic patients complain of fever, dry cough, sore throat and shortness of breath, while others present with non-specific symptoms like altered sense of smell or taste, myalgia, lethargy, diarrhoea and other gastrointestinal disturbances.

Though COVID-19 mainly affects the respiratory system, disease may cause widespread systemic and organ specific symptoms involving gastrointestinal, neurological, cardiovascular, immunological, cutaneous, hematological and coagulation systems. Minority of patients present with large vessel strokes, rare neurological syndromes like Guillain-Barre syndrome and Miller Fisher syndrome, which may be the sole manifestation of the disease. Some of the clinical presentations and pathogenesis of COVID are yet to be unraveled.
\end{abstract}

Key words: Covid-19, SARS-CoV-2, clinical characteristics

\section{Introduction}

An outbreak of pneumonia due to an unknown cause, originated in the city of Wuhan, Hubei Province of China, was first reported to the WHO Country Office, on $31^{\text {st }}$ December 2019 by the Chinese Health Authority. A novel beta coronavirus was discovered through the use of unbiased sequencing of the lower respiratory samples from patients with pneumonia ${ }^{1}$ and subsequently named as severe acute respiratory syndrome coronavirus 2 (SARS-CoV-2). The disease was designated as Coronavirus disease 2019 (COVID-19), by $\mathrm{WHO}^{2}$. Due to the high infectivity of the virus and person to person transmission, there was an exponential rise in number of cases in China and many other countries.

\section{Transmission}

SARS-CoV-2 is a single-stranded, positive sense RNA virus, in the family of Coronaviridae. The virus has spiked glycoproteins on the envelope resembling a crown. B lineage of beta-corona virus causes SARSCoV-1 and SARS-CoV-2 (COVID-19) infections ${ }^{3}$. A zoonotic transmission was suggested as three initially affected patients either worked at or frequent visitors to the local seafood market where live trade in of wild animals occurred ${ }^{1}$. Initial assessment of the transmission dynamics and epidemiologic characteristics of the disease indicated that epidemic has been growing as a result of human-to-human transmission ${ }^{4}$. According to the study published by Wu et al. (2020), the mean R0 of COVID-19 is 2.68 (95\% Cl: 2.47-2.86), meaning that on average each patient has been spreading the infection to 2.6 other people ${ }^{5}$. SARSCoV2 virus replication occurs in the upper respiratory tract and nasopharyngeal swab is shown to be positive up to 17 days and longer in COVID-19 patients with respiratory symptoms ${ }^{6}$. Two main modes of person to person transmission of SARS-CoV-2 is through respiratory droplets and contact with contaminated surfaces. It may be aerosolized during certain medical procedures such as intubation or the use of nebulizers. There are reports of COVID-19 positive individuals who are asymptomatic or with mild upper respiratory tract symptoms, suggesting the potential for asymptomatic or pre-symptomatic transmission. An increasing number of outbreaks of familial and institutional

\footnotetext{
${ }^{1}$ Teaching Hospital, Peradeniya, ${ }^{2}$ National Hospital, Kandy, Sri Lanka.

Correspondence: NMH, e-mail: niro_madhu@yahoo.com.sg
}

http://orcid.org/0000-0001-9494-0949

Received 01 May 2020, accepted 21 May 2020.

This is an open-access article distributed under the terms of the Creative Commons Attribution License, which permits unrestricted use, distribution, and reproduction in any medium, provided the original author and source are credited. 
transmission has raised the possibility of propagation of infection by asymptomatic individuals ${ }^{7,8}$.

\section{Incubation period and epidemiological data}

Incubation period of Covid-19 varies from 2-14 days. Most patients show clinical symptoms in 3-7 days. The median incubation period was 5.1 days (IQR 2-7) ${ }^{9}$.

Median age of the patients was in the range of 35-67 years. Men (49\%-73\%) are more affected than women $(27 \%-51 \%)^{10}$. According to a study by $\mathrm{Wu}$ Zunyou et al. (2020), Most cases (87\%) were between $30-79$ years, $8 \%$ were between $20-29$ years. There was only $3 \%$ in more than 80 years of age. There was $1 \%$ between $10-19$ years of age, and $1 \%$ was less than 10 years $^{10}$.

\section{Risk factors}

Male sex, older age (>65 years), smoking, obesity, diabetes, chronic pulmonary diseases, hypertension, cardiovascular diseases and chronic kidney disease are considered as poor epidemiological prognostic risk factors. The disease is severe when more risk factors are seen at presentation².

In a study by Chen et al. (2020) which included 99 patients with 2019-nCoV, 91 (48\%) patients had a comorbidity, with hypertension being the most common, followed by diabetes and coronary heart disease. From the overall population, $23.7 \%$ had at least one coexisting illness. Immunosuppression, cancer, and uncontrolled human immunodeficiency virus (HIV) infection may also confer an increased risk ${ }^{11}$.

\section{Case fatality rate}

The case fatality rate (CFR) vary between $2.3 \%$ and $5 \%$. No deaths were seen in the group aged 9 years and younger, but 8.0\% CFR noted in those aged 70 to 79 years. Patients who are 80 years and older had a $14.8 \% \mathrm{CFR}^{2}$. In critical cases CFR was $49.0 \%$. Death occurred within 1-2 weeks after ICU admission ${ }^{11}$. There were no deaths among mild and severe cases $^{12}$.

\section{Clinical profile}

There is a wide array of clinical features in patients infected with SARS-CoV-2. Depending on the clinical features, patients are generally divided as mild, severe and critical. Although most exhibit a mild illness (81\%), $14 \%$ have severe and 5\% have critical illness ${ }^{2}$. Approximately $10 \%$ will require hospital admission due to COVID-19 pneumonia, of which approximately $10 \%$ will require ICU care ${ }^{11}$. The usual triad of symptoms at the time of presentation are fever, dry cough and dyspnoea. In several studies done on Chinese patients, fever, cough and shortness of breath were the most prominent symptoms. Fever was present on admission in $43.8 \%-83 \%$ of patients while $88.7 \%$ has developed fever during hospitalization. The second most common symptom was cough (31\%-94.8\%). Dyspnoea varied from $6.9 \%$ to $63.5 \% \%^{2,11,13-18}$. Dyspnoea developed after a median of 5 to 8 days of occurrence of initial symptoms in some case series and it was suggestive of worsening disease ${ }^{16}$. According to a US study by Agarwal et al. (2020), the most common presenting symptoms were fever (94\%), cough (88\%), and dyspnoea (81\%). Loss of smell and taste sensations were reported by three (19\%) patients. Same study showed low oxygen saturation in $38 \%$ of patients, whilst $31 \%$ were hypotensive on admission. Sepsis was the most frequently observed complication, followed by respiratory failure, ARDS, heart failure and septic shock $^{17}$.

Other symptoms reported in Chinese studies include fatigue (23\%-38.1\%), sputum production $(33.4 \%)$, shortness of breath $(18.6 \%)$, sore throat $(13.9 \%)$, and headache $(6.5 \%-13.6 \%)^{3}$. Nausea, vomiting (4\%-5.0\%), diarrhoea (3.0\%-5\%) $)^{12-16}$, conjunctivitis, anosmia and ageusia were less common. Cardiac complications, including new or worsening heart failure, arrhythmias, or myocardial infarction were seen ${ }^{11}$. Elderly, immunosuppressed and pregnant patients may present with atypical symptoms.

In a European study done on 1420 patients with COVID-19, 420 patients completed the study (962 women, $30.7 \%$ were health care workers). The mean age of patients of that cohort was $39.17 \pm 12.09$ years. The most common symptoms were headache (70.3\%), loss of smell (anosmia) (70.2\%), nasal obstruction $(67.8 \%)$, cough $(63.2 \%)$, asthenia (63.3\%), myalgia $(62.5 \%)$, rhinorrhea $(60.1 \%)$, gustatory dysfunction (54.2\%) and sore throat (52.9\%). Fever was reported by only $45.4 \% \%^{18}$.

\section{Gastrointestinal manifestations}

In a retrospective, single-center study of 84 patients with SARS-CoV-2 pneumonia in Wuhan Union Hospital, China, revealed that $31 \%$ of patients had diarrhoea ${ }^{19}$. A significantly higher proportion of patients presented with diarrhoea had virus RNA in stool than patients without diarrhoea. The study concluded that elimination of SARS-CoV-2 from stool takes longer than 
elimination from the nose and throat. Study compared clinical symptoms in diarrhoeal and non-diarrhoeal group. The COVID-19 patients with diarrhoea had headache, myalgia or fatigue, cough, sputum production, nausea, vomiting more frequently than those patients without diarrhoea $(p<0.01)$. Abdominal pain, abdominal distension and tenesmus were occasional findings. The duration of fever and dyspnoea in patients with diarrhoea was significantly longer than those without diarrhoea $(P<.05)$. The characteristics of diarrhoea include increased defecation frequency (3 14 times per day), stool with no mucous, pus or blood. The diarrhoea symptom may be caused by the invaded ACE2-expressing enterocytes. The underlying molecular pathogenesis needs to be further investigated ${ }^{19}$.

In another study which included 116 patients from California ${ }^{20}$, gastrointestinal symptoms were reported by $31.9 \%$ of patients, out of which $89.2 \%$ showed mild symptoms. Loss of appetite (22.3\%), nausea/vomiting (12.0\%) and diarrhoea (12.0\%) were the most common symptoms. Gastrointestinal symptoms did not occur in isolation or as an initial manifestation of SARS-CoV2 infection. Median duration of gastrointestinal-specific symptoms including nausea/vomiting or diarrhoea was 1 day [IQR 0-4]; which was significantly shorter than the duration of respiratory symptoms $(P<0.001)$. Out of 65 patients who had liver enzymes measured 26 patients, (40\%) demonstrated hepatic dysfunction with a slightly higher levels of aspartate aminotransferase (AST) compared to alanine aminotransferase $(A L T)^{20}$.

\section{Neurological manifestations}

Corona viruses may invade the nervous system, the route of neuro-propagation from the nasal cavity to the olfactory bulb and to the brain stem has been demonstrated in animal models ${ }^{21}$. Furthermore, in human respiratory tract, nasal epithelial cells display the highest expression of the angiotensin-converting enzyme 2 (ACE-2) receptors for SARS-CoV-222.

Altered sense of smell and taste is recognized as a common manifestation of COVID-19. In a crosssectional survey of 202 mildly symptomatic COVID19 patients in Italy, any altered sense of smell or taste was reported by 130 patients $(64.4 \%, 95 \% \mathrm{Cl}, 57.3 \%$ $71.0 \%)^{23}$. The timing of an altered sense of smell or taste in relation to onset of other symptoms was; before the onset in $11.9 \%$, at same time in $22.8 \%$, and after other symptoms in $26.7 \%$. An altered sense of smell or taste was reported as the only symptom in $3 \%$ of patients ${ }^{23}$. However, according to the data from other European centres, the prevalence of altered sensation of smell and taste vary between $34 \%$ to $88 \%$ of hospitalized COVID-19 patients ${ }^{18,24}$.

Mao et al. analyzed the frequency of neurological manifestations in 214 hospitalized patients with laboratory confirmed diagnosis of SARS-CoV-2 infection from three designated COVID care hospitals in Wuhan, China $^{25}$. It was shown that 78 (36.4\%) patients had neurologic manifestations; central nervous system (CNS) symptoms in $24.8 \%$ (headache, dizziness, impaired consciousness, ataxia, acute cerebrovascular disease, and epilepsy), peripheral nervous system (PNS) symptoms in 8.9\% (hypogeusia, hyposmia, hypopsia, and neuralgia), and skeletal muscular symptoms in $10.7 \%$. In patients with CNS symptoms, the most common complaints were dizziness (16.8\%) and headache $(13.1 \%)$ and, in patients with PNS symptoms, anosmia in $5.1 \%$ patients and ageusia in $5.6 \%$ patients $^{25}$.

Patients with severe illness can present with seizures, altered level of consciousness and confusion. As there are several confounding factors which might affect the clinical presentation and sensorium of patients with severe COVID-19 like hypoxia, multi-organ failure, metabolic and electrolyte derangements, medications and therapeutic interventions ${ }^{26}$, it is difficult to analyze them as a direct effect of COVID-19.

Large vessel stroke has been a significant presenting symptom in young COVID-19 patients. Five cases of large-vessel stroke, with the mean NIHSS score of 17, were reported in patients younger than 50 years from New York, USA. They had hemiplegia, facial palsy, dysarthria, gaze palsies and four of them had reduced level of consciousness on admission. Two of them had mild COVID-19 symptoms like cough, fever and lethargy ${ }^{27}$. A retrospective study of data from the COVID-19 outbreak in Wuhan, China, showed that the incidence of stroke among hospitalized patients with Covid-19 was approximately 5\%; the youngest patient in that series was 55 years of age ${ }^{28}$.

Few diagnosed cases have reported with GuillainBarré syndrome (GBS) as a presenting feature of COVID-19. Toscano et al. reported four patients in their case series diagnosed with GBS having positive PCR test for SARS-CoV-2. The first symptoms of GBS were lower-limb weakness and paresthesia in four patients and facial diplegia followed by ataxia and paresthesia 
in one patient. The interval between the onset of symptoms of COVID-19 and the first symptoms of GBS ranged from 5 to 10 days ${ }^{29}$. Critical illness neuropathy and myopathy usually develops later in the course of critical illness and it should be distinguished from GBS in COVID-19 which appears rather early phase of the disease.

Gutiérrez-Ortiz et al. have described occurrence of Miller Fisher syndrome and polyneuritis cranialis as unusual initial neurological manifestations of COVID-1930. Miller Fisher syndrome was characterized by the acute onset of external ophthalmoplegia, ataxia, and loss of tendon reflexes. Patient was treated with intravenous immunoglobulin and he had a full recovery within 2 weeks.

There are few case reports mentioning the occurrence of odd combination of neurological symptoms like ophthalmoparesis, leg paresthesia and areflexia in some of the COVID-19 patients ${ }^{31}$.

\section{Haematological manifestations}

\section{Thrombocytopenia}

Haematological manifestations in COVID-19 include thrombocytopenia and thrombotic complications. Mild thrombocytopenia is a common finding with the platelet count ranging from $100-150 \times 10^{9} / L^{32}$. The pooled analysis revealed that platelet count was significantly lower in patients with more severe COVID-19, therefore it may serve as a clinical indicator of worsening illness during hospitalization ${ }^{33}$. The mechanism for thrombocytopenia in SARS-CoV2 is multifactorial $^{32}$. A SARS CoV-2 positive patient diagnosed as immune thrombocytopenic purpura who recovered after immunoglobulin therapy suggests a possible autoimmune response as a cause for thrombocytopenia ${ }^{34}$.

\section{Thrombotic complications}

COVID-19 may predispose patients to thrombotic disease, both in the venous and arterial circulations, due to excessive inflammation, platelet activation, endothelial dysfunction, and stasis ${ }^{35}$. The risk of thromboembolism was higher in patients with severe disease who were treated at Intensive Care Unit (ICU). There were several reports of thrombotic complications such as central venous catheter associated thrombosis, deep venous thrombosis (DVT) and pulmonary embolism $(P E)^{36}$.
In a cohort of 81 ICU patients in China, where routine thromboprophylaxis was not used, the proportion of DVT was $25 \%{ }^{37}$. In a study done on 184 ICU patients in a Dutch hospital where DVT prophylaxis is used incidence of DVT was $37 \%^{38}$. Similar observations were seen in two studies done in France and Italy ${ }^{39,40}$. Similarly, Spiezia et al. (2020) observed severe hypercoagulability rather than a consumptive coagulopathy ${ }^{41}$.

Laboratory findings of patients with COVID-19 who had developed thrombotic complications include elevated D-dimer levels, prolonged prothrombin time and fibrinogen levels ${ }^{36,39}$. Patients who died from COVID19 had progressively rising D-Dimer levels than who survived ${ }^{42}$. Patients with D-Dimers more than $1 \mathrm{mg} / \mathrm{L}$ had 18-fold increased risk of death ${ }^{43}$.

\section{Cutaneous manifestations}

Though cutaneous manifestations are not well described in COVID-19, it is important to identify as they may manifest before the respiratory symptoms. In an Italian study, out of 88 patients 18 patients (20.4\%) developed cutaneous manifestations; erythematous rash (14 patients), widespread urticaria (3 patients), and chicken pox like lesions (1 patient $)^{44}$.

Sachdeva et al, described eighteen articles and three additional cases in a review. The most common cutaneous manifestation of COVID-19 was found to be maculopapular exanthem (morbilliform), presenting in $36.1 \%$ (26/72) patients. The other cutaneous manifestations included: a papulovesicular rash, urticaria, painful acral red purple papules, livedo reticularis lesions and petechiae. Majority of lesions were localized on the trunk ${ }^{45}$.

Vasculitic skin changes including petechiae, purpura, ecchymosis, livedoid lesions have been described mostly in paediatric COVID-1946. Veena $\mathrm{G}$ et al. reported a case history of a 6-month-old baby diagnosed of COVID-19 and Kawasaki Disease who presented with clinical features such as fever, limbic sparing conjunctivitis, prominent tongue papilla, a polymorphous, maculopapular rash, and swelling of the hands and lower extremities ${ }^{47}$.

\section{Conclusion}

In conclusion, COVID-19, causes widespread involvement in many organs and systems. Though the classic triad of symptoms comprising fever, dry cough and dyspnoea are usually present, unusual presen- 
tations involving many systems have been noted in variable number of patients. Due to its novelty and highly infectious nature, many aspects of COVID-19 are yet to be unraveled. It will be a daunting task ahead in containing the disease, as well as in managing the patients.

\section{References}

1. Zhu N, Zhang D, Wang W, et al. A Novel Coronavirus from Patients with Pneumonia in China, 2019 New England Journal of Medicine 2020; 382: 727-33. DOI: 10.1056/NEJMoa 2001017

2. Akriti S, Altamash S, Singh R, Singh AK. Covid-19: From bench to bed side. Diabetes \& Metabolic Syndrome: Clinical Research and Reviews 2020; 14(4): 277-81.

3. Chen Y, Liu Q, Guo D. Emerging coronaviruses: genome structure, replication, and pathogenesis. Journal of Medical Virology 2020; 92(4): 418-23.

4. Li Q, Guan X, Wu P, et al. Early transmission dynamics in Wuhan, China, of novel coronavirus-infected pneumonia. New England Journal of Medicine 2020; 382: 1199-207. DOI: 10.1056/NEJMoa2001316

5. Wu JT, Leung K, Leung GM. Nowcasting and forecasting the potential domestic and international spread of the 2019nCoV outbreak originating in Wuhan, China: a modeling study Lancet 2020; 395: 689-79.

6. Yang R, Xien G, Yong X. Patients with respiratory symptoms are at greater risk of COVID-19 transmission Respiratory Medicine. 2020. 165. Article 05935 doi: 10.1016/j.rmed. 2020.105935

7. Ye F, Xu S, Rong Z, et al. Delivery of infection from asymptomatic carriers of COVID-19 in a familial cluster. International Journal of Infectious Diseases. 2020; 94 : 133-38.

8. Bai Y, Yao L, Wei T, et al. Presumed Asymptomatic Carrier Transmission of COVID-19. Journal of American Medical Association 2020; 323(14): 1406-7. doi:10.1001/jama. 2020.2565

9. Lauer SA, Grantz KH. Qifang B, et al. The incubation period of coronavirus disease 2019 (covid-19) from publicly reported confirmed cases: estimation and application. Annals of Internal Medicine 2020. https://doi.org/10.7326/M20-050

10. Wu Z, McGoogan JM. Characteristics of and Important Lessons from the Coronavirus Disease 2019 (COVID-19) Outbreak in China: Summary of a Report of 72314 Cases from the Chinese Center for Disease Control and Prevention Journal of American Medical Association 2020; 323: 1243-45.

11. Chen N, Zhou M, Dong Z, et al. Epidemiological and clinical characteristics of 99 cases of 2019 novel coronavirus pneumonia in Wuhan, China a descriptive study; Lancet
2020; 395: 507-13. https://doi.org/10.1016/ S01406736(20)30211-7

12. Yang $X, Y u Y, X u J$, et al. Clinical course and outcomes of critically ill patients with SARS-CoV-2 pneumonia in Wuhan, China: a single-centered, retrospective, observational study. Lancet 2020. (published online February 21,2020 ) https:// doi.org/10.1016/S2213-2600(20)30079-5

13. Fei Zhou, Ting Yu, Ronghui Du, et al. Clinical course and risk factors for mortality of adult patients with Covid 19 in Wuhan China, a retrospective cohort study. Lancet 2020; (published online March 9, 2020) https://doi.org/10.1016/S01406736(20)30566-3

14. Huang $C$, Wang $Y$, Ren L, et al. Clinical features of patients infected with 2019 novel coronavirus in Wuhan, China; Lancet 2020; 395: 497-506. https://doi.org/10.1016/ S01406736(20)30183-5

15. Guan W, Ni Z, Hu Yu, et al. Clinical Characteristics of Coronavirus Disease 2019 in China. New England Journal of Medicine (published online February 28, 2020), doi: 10.1056/nejmoa2002032

16. Gandhi RT, Lynch JB, del Rio C. Mild or Moderate Covid-19 New England Journal of Medicine 2020 DOI: 10.1056/ NEJMcp2009249

17. Aggarwal S, Garcia-Telles N, et al. Clinical features, laboratory characteristics, and outcomes of patients hospitalized with coronavirus disease 2019 (COVID-19): Early report from the United States Diagnosis (Berl) 2020; 7(2): 91-96. doi: 10.1515/dx-2020-0046.

18. Lechien JR, Chiesa-Estomba CM. Clinical and Epidemiological Characteristics of 1,420 European Patients with mild-tomoderate Coronavirus Disease. Journal of Internal Medicine 2020. https://doi.org/10.1111/joim.13089

19. Wei X, Wang X, Niu Y, et al. Diarrhea is associated with prolonged symptoms and viral carriage in COVID-19. Clinical Gastroenterology and Hepatology 2020; (In press). https:// doi.org/10.1016/j.cgh.2020.04.030

20. Cholankeril G, Podboy A, Aivaliotis V I et al. High Prevalence of Concurrent Gastrointestinal Manifestations in Patients with SARS-CoV-2: Early Experience from California, Gastroenterology 2020, (In press), doi: https://doi.org/ 10.1053/ j.gastro.2020.04.008.

21. Dubé M, Le CoupanecA, Wong AHM, Rini JM, Desforges M, Talbot PJ. Axonal transport enables neuron-to-neuron propagation of human coronavirus OC43. Journal of Virology 2018. 92:e00404-18. https://doi.org/10.1128/JVI .00404-18

22. Sungnak W, Huang $N$, Bécavin $C$, Berg $M$, Network HLB. SARS-CoV-2 entry genes are most highly expressed in nasal goblet and ciliated cells within human airways. ArXiv 200306122 2020. https://arxiv.org/abs/2003.06122

23. Spinato G, Fabbris C, Polesel J, et al. Alterations in Smell or Taste in Mildly Symptomatic Outpatients With SARS-CoV-2 Infection. Journal of American Medical Association 2020 (Published on line 22 April 2020) doi:10.1001/jama.2020.6771 
24. Lechien JR, Chiesa-Estomba CM, De Siati DR, et al. Olfactory and gustatory dysfunctions as a clinical presentation of mild-to-moderate forms of the coronavirus disease (COVID-19): a multicenter European study. European Archives of Oto-Rhino-Laryngology 2020 (published on line 06 April 2020) https://doi.org/10.1007/ s00405-020-05965-1

25. Mao L, Jin $\mathrm{H}$, Wang $\mathrm{M}$, et al. Neurological Manifestations of Hospitalized Patients with COVID-19 in Wuhan, China. JAMA Neurology 2020. (published online April 10, 2020) doi:10.1001/ jamaneurol.2020.112

26. Asadi-Pooyaa AA, Leila S. Central nervous system manifestations of COVID-19: A systematic review. Journal of Neurology and Science 2020; 413: 116832. https://doi.org/ 10.1016/j.jns.2020.116832

27. Oxley T J, Mocco J, Majidi S, et al. Large-Vessel Stroke as a Presenting Feature of Covid-19 in the Young. New England Journal of Medicine 2020 (published on line April 2020) DOI: 10.1056/NEJMc2009787

28. Li Y, Wang M, Zhou Y, et al. Acute cerebrovascular disease following COVID-19: a single center, retrospective, observational study. New England Journal of Medicine 2020: 282: 20 DOI: 10.1056/NEJMc2009787

29. Toscano G, Palmerini F, Ravaglia S, et al. Guillain-Barré Syndrome Associated with SARS-CoV-2. New England Journal of Medicine 2020 DOI: 10.1056/NEJMc2009191 [Online ahead of print].

30. Gutiérrez-Ortiz C, Méndez A, Rodrigo-Rey S, et al. Miller Fisher Syndrome and polyneuritis cranialis in COVID-19 Neurology 2020: DOI: 10.1212/WNL.0000000000009619

31. Dinkin M, Gao B, Kahan J, et al. COVID-19 presenting with ophthalmoparesis from cranial nerve palsy. Neurology. May 2020, 10.1212/WNL.0000000000009700; DOI: 10.1212/ WNL.0000000000009700

32. Lippi G, Plebani M, Michael Henry B. Thrombocytopenia is associated with severe coronavirus disease 2019 (COVID19) infections: A meta-analysis. Clinica Chimita Acta 2020 Mar 13; 506:145-148. doi: 10.1016/j.cca.2020.03.022.

33. Yang $\mathrm{M}, \mathrm{Ng} \mathrm{MHL}$, Li CK. Thrombocytopenia in patients with severe acute respiratory syndrome. Hematology 2005; 10 : 101-5.

34. Zulfi A, Lorenzo-Villalba N, Hassler P, Andres E. Immune Thrombocytopenic Purpura in a Patient with Covid-19. New England Journal of Medicine 2020 Apr 30; 382(18):e43. doi: 10.1056/NEJMc2010472

35. Bikdeli, B, Madhavan MV, Jimenez D, et al, COVID-19 and Thrombotic or Thromboembolic Disease: Implications for Prevention, Antithrombotic Therapy, and Follow-up. Journal of the American College of Cardiology 2020 (published online 17 April, 2020) https://doi.org/10.1016/j.jacc. 2020.04.031
36. Middeldorp S, Coppens M, van Haaps TF, et al, (2020), Incidence of venous thromboembolism in hospitalized patients with COVID-19. Journal of Thrombosis and Haemostasis 2020 (published online 05 May, 2020) doi:10.1111/jth.14888

37. Cui S, Chen S, Li X, Liu S, Wang F. Prevalence of venous thromboembolism in patients with severe novel coronavirus pneumonia. Journal of Thrombosis and Haemostasis 2020 (published online 2020 April 09) https://doi.org/10.1111/ jth.14830

38. Klok FA, Kruip MJHA, van der Meer NJM, et al. Incidence of thrombotic complications in critically ill ICU patients with COVID-19 Thrombosis Research. 2020; S00493848(20)30120-1.(published online Apr 10, 2020]. doi:10.1016/j.thromres.2020.04.013

39. Helms J, Tacquard C, Severac F, et al. High risk of thrombosis in patients with severe SARS-CoV-2 infection: a multicenter prospective cohort study. Intensive Care Medicine 2020 https://doi.org/10.1007/s00134-020-06062

40. Lodigiani C, lapichino G, Carenzo L, et al. Venous and arterial thromboembolic complications in COVID-19 patients admitted to an academic hospital in Milan, Italy. Thrombosis Research 2020; 191: 9-14. (published online, 2020 Apr 23) doi:10.1016/ j.thromres.2020.04.024

41. Spiezia L, Boscolo A, Poletto F, et al. COVID-19-Related Severe Hypercoagulability in Patients Admitted to Intensive Care Unit for Acute Respiratory Failure. Thrombosis and Haemostasis 2020 (published online: 2020 April 21) doi:10.1055/s-0040-1710018

42. Wang D, Hu B, Hu C, et al. Clinical Characteristics of 138 Hospitalized Patients With 2019 Novel Coronavirus-Infected Pneumonia in Wuhan, China. Journal of American Medical Association 2020; 323(11): 1061-9. doi:10.1001/jama. 2020.1585

43. Weiss $P$, Murdoch DR. Clinical course and mortality risk of severe COVID-19. Lancet 2020; 395(10229): 1014-15. doi:10.1016/S0140-6736(20)30633-4

44. Recalcati, S. Cutaneous Manifestations in COVID-19: A First Perspective. Journal of the European Academy of Dermatology and Venereology 2020. (published online March 26, 2020)

45. Sachdeva M, Gianotti R, Shah M, et al. Cutaneous manifestations of COVID-19: Report of three cases and a review of literature. Journal of Dermatological Science 2020 doi:10.1016/j.jdermsci.2020.04.011

46. TüRsen Ü, Türsen B, Lotti, T. Coronavirus-days in dermatology. Dermatologic Therapy 2020. (published online 15 April, 2020) doi:10.1111/dth.13421

47. Jones VG, Mills M, Suarez D, et al. COVID-19 and Kawasaki Disease: Novel Virus and Novel Case. Hospital Pediatrics 2020, hpeds.2020-0123; DOI: 10.1542/hpeds.2020-0123 